\title{
A Measure of the Incongruity Hypothesis: Effects of Two Orienting Tasks on Memory for Targets of Categorical and Orthographic Distinctiveness
}

\author{
Brooke P. Ludwig ${ }^{1}$, Tracy B. Henley ${ }^{1} \&$ Raymond J. Green ${ }^{1}$ \\ ${ }^{1}$ Department of Psychology, Texas A\&M University-Commerce, Commerce, USA \\ Correspondence: Tracy B. Henley, Department of Psychology, Counseling and Special Education, Texas A \& M \\ University - Commerce, Commerce, TX 75429, USA. E-mail: thenley@tamu-commerce.edu
}

Received: March 27, 2012

Accepted: May 4, 2012 Published: June 1, 2012

doi:10.5539/ijps.v4n2p88

URL: http://dx.doi.org/10.5539/ijps.v4n2p88

\begin{abstract}
This study examined the effects of two orienting tasks - pleasantness-judging and syllable counting-on memory for two lists of words containing either categorically distinctive targets or orthographically distinctive targets. Analysis of the number of filler words recalled suggested that there was a significant difference between the two word lists, but not between the two orienting tasks. There was also a significant interaction effect between list and task on recall for filler words. However, analysis of the number of target words recalled revealed no significant difference between the two lists or the two tasks. As such, some of the results of this study were inconsistent with Schmidt's (1991) Incongruity Hypothesis.
\end{abstract}

Keywords: memory, distinctiveness, incongruity hypothesis

\section{Introduction}

The relationship between the structural organization of material, such as a word list, and the memory of that material has long been of interest for psychology (e.g., Von Restorff, 1933). In terms of the way information is processed, stimuli that are unique, bizarre, or otherwise distinctive tend to attract more attention than things that are considered more common (e.g., Wallace, 1965). This study examined Schmidt's (1991; 1996) Incongruity Hypothesis regarding the effects of target items that represent either primary (categorical) or secondary (orthographic) distinctiveness.

Classic cognitive research concerning the effects of distinctive events on memory (e.g., Bruce \& Gaines, 1976; Hunt \& Mitchell, 1982; Schmidt, 1985; Watkins \& Watkins, 1975) has established that fundamental processes such as the ability to recall, recognize, and organize information are influenced by the presence of distinctive targets. More specifically, Schmidt's (1991) Incongruity Hypothesis proposes that distinctiveness affects memory performance during three phases of processing. The first phase is the attentional response, while the second phase involves numerous control processes such as elaboration, relational processing, and rehearsal. The third phase is the memory test, where the effects of distinctiveness depend on the degree to which stored information is able to support memory performance. According to Schmidt, the effects of the processes during phase two are especially important because they may serve to increase or decrease the impact of distinctiveness.

Schmidt (1991) also identified two classes of distinctiveness. The first class, primary distinctiveness, contains items that stand out because one or more elements in the material do not fit the overall structure activated by the to-be-remembered material. Stated another way, this class involves categorical or semantic distinctiveness. In contrast, secondarily distinctive items stand out because they are atypical members of a conceptual category, or they describe semantic relations between concepts that are atypical of the fundamental concepts i.e., they possess orthographic distinctiveness.

Schmidt (1991) further explains that the effects of primary distinctiveness are more pronounced in recall than recognition because the distinctive items share physical or conceptual features that lead to relational processing (his phase two) and clustering in recall (his phase three). In contrast, secondary distinctiveness does not lead to clustering of distinctive items (e.g., Hunt \& Mitchell, 1982), presumptively because such items do not share similar features, and so there is no basis for relational processing.

One well established technique for studying memory involves analyzing the effects of various orienting tasks on 
the retention of words (e.g., Bird \& Campbell, 1982). Orienting tasks are especially useful because they provide a degree of control over encoding by directing attention to particular word features. Indeed, the classic research concerning the effects of orienting tasks on free recall and organization has produced several conclusions. For example, a number of studies have shown that semantic orienting tasks that encourage participants to focus on the meaning of the stimulus items leads to greater recall than non-semantic orienting tasks that direct participants to focus on the surface structure of the stimulus item (e.g., Craik \& Tulving, 1975) Additionally, Jenkins and colleagues (e.g., Hyde \& Jenkins,1969; Johnston \& Jenkins, 1971; Till \& Jenkins, 1973; Walsh \& Jenkins, 1973) have also demonstrated that orienting tasks can be used to influence the amount and organization of free recall, as well as showing that semantic orienting tasks lead to greater clustering and recall of stimulus items than non-semantic orienting tasks.

The present study used a semantic (pleasantness-judging) and a non-semantic (syllable counting) orienting task to direct attention, and recall tests for two lists of words that contained target items that were either categorically or orthographically distinctive as a way to measure the degree to which stored information is able to support optimal memory performance as discussed by Schmidt (1991). Specifically, one aim of this study was to determine if the "control processes" at phase two of Schmidt's model (which are said to increase or decrease the effects of distinctiveness) can be manipulated using semantic and non-semantic orienting tasks. A second aim was to determine whether the semantic and non-semantic orienting tasks would have different effects on categorically and orthographically distinctive targets.

\section{Method}

\subsection{Participants and Materials}

Eighty undergraduate students from a university in Texas participated in the experiment. All participants were randomly drawn from a pool of potential volunteers solicited by the Psychology Department. Formal demographics were not obtained for this study, but the participant pool was $70 \%$ Caucasian (with African-Americans and Hispanics accounting for another 26\%), 61\% female, and had a mean age of 26 and a median age of 22 .

The equipment and materials used for this study included a desk-top IBM personal computer connected to an Epson computer projector system, one Microsoft PowerPoint presentation of three (twenty item) word lists, and individual response booklets.

The first word list was a practice set that contained 20 items, the second list had four categorically distinctive word items embedded in a list of 20 items from a different conceptual category, and the last list had four orthographically distinctive words embedded in a list of 20 orthographically common words from the same category.

The categorically distinctive list and the orthographically distinctive list were adapted from Hunt and Mitchell (1982). For the primary distinctiveness condition, the categorically distinctive word list consisted of 16 common musical instruments, with four animal items embedded in positions 9-12. For the secondary distinctiveness condition, the orthographically distinctive word list consisted of 16 common animals with four orthographically distinctive animal items embedded in positions 9-12. The two classes of target items were matched with each other and with filler items on frequency of occurrence and imagery ratings, and differed only on the dimension of orthography.

\subsection{Procedure}

Prior to the experiment, participants were randomly assigned to one of four different treatment conditions based on the combination of two levels of distinctiveness and two different orienting tasks. The two levels of distinctiveness were category distinctiveness and orthographic distinctiveness. The two orienting tasks were pleasantness-judging and syllable counting. The pleasantness-judging group was instructed to judge the pleasantness of each word in the stimulus list using long-established methods (e.g., Bird \& Campbell, 1982; Hyde \& Jenkins, 1969; Till \& Jenkins, 1973) on a scale of 1 to 5 (1 indicating the least pleasant and 5 indicating the most pleasant). The syllable counting group was instructed to write down the number of syllables for each word in the stimulus list also using long-established methods (e.g., Bird \& Campbell, 1982; Elmes \& Thompson, 1977; Walsh \& Jenkins, 1973). For each orienting task, one group of subjects was tested on a word list containing categorically distinctive targets and one group of subjects was tested on a word list containing orthographically distinctive targets.

All participants were seated in a classroom, given specific instruction regarding the orienting task, and informed that they would be tested on their recall for a list of words. The materials were presented via PowerPoint onto a 
large projection screen well-suited for the room and the task. Following Hunt \& Mitchell (1982), the words were presented individually on slides at a 5 -second exposure rate. An example was given so that the participants could familiarize themselves with this procedure. After the list presentation, 3 minutes were allowed for free recall. On the recall test, participants were asked to write down the words on their answer sheet in the same order that they remembered them.

A contiguous recall score was determined for participants that recalled at least two target items. The contiguous recall score was determined as follows: those who had no contiguous recall of target items (i.e., 0/2, 0/3, or 0/4) received a score of " 0 ," those who recalled two target items contiguously (i.e., $2 / 2,2 / 3$, or $2 / 4$ ) received a score of " 2 ," those who recalled three target items contiguously (i.e., 3/4, or 3/3) received a score of " 3 ," and those who recalled all four target items contiguously received a score of "4."

\subsection{Data Analysis}

The design of this experiment was a completely randomized 2 X 2 factorial ANOVA. The independent variables were type of distinctiveness (categorical or orthographic) and type of orienting task (pleasantness-judging or syllable-counting). All factors were manipulated between subjects. Three dependent measures were considered for this study: the number of background items recalled, the number of target items recalled, and the number of target items listed contiguously in recall. A completely randomized two-factor ANOVA was used to analyze the number of background items recalled. Analysis of the data revealed that the remaining dependent variables, recall of target words and contiguous recall of target words, did not meet the normality or equality of variance assumptions required by the ANOVA model. Therefore, the dependent variables recall of target words and contiguous recall of target words were each combined with the independent variables "type of list" and "type of task" to produce four separate non-parametric analyses using the SPSS Kruskal-Wallis procedure.

\section{Results}

Data screening prior to analysis revealed that there was one outlier with a background recall score of zero. Boxplots showed that the spreads of the four groups were approximately symmetric and similar. Histograms also showed approximately normal distributions for all four groups. Lastly, the Levene statistic $(3.02, p=.035)$ indicated the four groups had equal variance.

Participants showed significantly greater recall of background words on the orthographically distinctive list (with a mean of 9.58) than on the categorically distinctive list (mean of 7.0 ), $F(1,76)=14.78, p<.0005$. In contrast, there was no significant difference in the number of filler words recalled between participants in the syllable-counting task group (with a mean of 7.7) and the pleasantness-judging task group (mean of 8.88). In addition, there was a significant interaction effect between list condition and task condition on recall of filler words, $F(1,76)=8.7, p=.004$.

The first Kruskal-Wallis analysis of the recall of target words revealed that there was no significant difference in the recall of target words between the orthographically distinctive list and the categorically distinctive list. The second analysis revealed that there was no significant difference in the recall of target words between the syllable-counting task group and the pleasantness-judging task group. The analysis of the contiguous recall of target words revealed that there was no significant difference in the contiguous recall of target words between the orthographically distinctive list and the categorically distinctive list. Last, there was no significant difference in the contiguous recall of target words between the syllable-counting task group and the pleasantness-judging task group.

\section{Discussion}

According to Schmidt's (1991) Incongruity Hypothesis, orthographically and categorically distinctive items are expected to be incongruent based on context. Target items are predicted to evoke an attentional response based on the contrast between these items and the other (filler) list items. Additionally, control processes operating at phase two in the model should serve to either increase or decrease the effects of distinctiveness. In previous studies (e.g., Bruce \& Gaines, 1976; Schmidt, 1985), the effects of category and orthographic distinctiveness have been found in both recognition and free recall. In the present study, a recall task was chosen so that the clustering of target items could be measured.

The results of this study were both consistent and inconsistent with Schmidt's (1991) Incongruity Hypothesis. As Schmidt would predict, memory for filler items was suppressed by the presence of categorically distinctive items on a recall test. Additionally, recall was affected by the combination of the semantic and non-semantic orienting tasks and distinctiveness of the word lists. Likewise, orthographically distinctive words were just as likely to be recalled as categorically distinctive words. 
Nevertheless, the results of this study were also inconsistent with Schmidt's Incongruity Hypothesis in several ways. For example, semantic and non-semantic orienting tasks had no effect on the recall of filler words, and semantic and non-semantic orienting tasks also had no effect on the recall of target words. It was also the case that semantic and non-semantic orienting tasks did not influence contiguous recall of target items, and that categorically distinctive words were no more likely to be recalled contiguously than orthographically distinctive words.

One possible explanation for these inconsistent results is that the pleasantness-judging task caused the categorically distinctive target items to stand out even more, while having the opposite effect on the orthographically distinctive target items. It is also possible that the syllable counting task caused the orthographically distinctive target items to stand out even more, and that it had the opposite effect on the categorically distinctive targets. And, it is possible that there was an interaction between list condition and task condition which could not be assessed with this data set given the need for non-parametric analysis. In conclusion, although these finding offer some support for the Incongruity Hypothesis, they also suggest that more research is needed before the complete details of the model can be articulated.

\section{Acknowledgement}

The authors would like to thank Dean Ginther and Devin Pierce for their contributions to this work.

\section{References}

Bird, C. P., \& Campbell, P. L. (1982). Orienting tasks and release from proactive inhibition. American Journal of Psychology, 95, 251-265. http://dx.doi.org/10.2307/1422469

Bruce, D., \& Gaines, M. T. (1976). Tests of an organizational hypothesis of isolation effects in free recall. Journal of Verbal Learning and Verbal Behavior, 15, 59-72. http://dx.doi.org/10.1016/S0022-5371(76)90007-4

Craik, F. I. M., \& Tulving, E. (1975). Depth of processing and the retention of words in episodic memory. Journal of Experimental Psychology: General, $104, \quad 268-294$. http://dx.doi.org/10.1037/0096-3445.104.3.268

Elmes, D. G., \& Thompson, J. B. (1977). Attenuating the effects of different levels of processing: The role of cue position and cue/word interval. Bulletin of the Psychonomic Society, 10, 152-154. Retrieved from http://psycnet.apa.org/psycinfo/1978-20245-001

Hunt, R. R., \& Mitchell, D. B. (1982). Independent effects of semantic and nonsemantic distinctiveness. Journal of Experimental Psychology: Human Learning \& Memory, 8, 81-87. http://dx.doi.org/10.1037/0278-7393.8.1.81

Hyde, T. S., \& Jenkins, J. J. (1969). The differential effects of incidental tasks on the organization of recall of a list of highly associated words. Journal of Experimental Psychology, 82, 472-481. http://dx.doi.org/10.1037/h0028372

Johnston, C. D., \& Jenkins, J. J. (1971). Two more incidental tasks that differentially affect associative clustering in recall. Journal of Experimental Psychology, 89, 92-95. http://dx.doi.org/10.1037/h0031184

Schmidt, S. R. (1985). Encoding and retrieval processes in the memory for conceptually distinctive events. Journal of Experimental Psychology: Learning, Memory, \& Cognition, 11, 565-578. http://dx.doi.org/10.1037/0278-7393.11.3.565

Schmidt, S. R. (1991). Can we have a distinctive theory of memory? Memory \& Cognition, 19, 523-542. http://dx.doi.org/10.3758/BF03197149

Schmidt, S. R. (1996). Category typicality effects in episodic memory: Testing models of distinctiveness. Memory \& Cognition, 24, 595-607. http://dx.doi.org/10.3758/BF03201086

Till, R. E., \& Jenkins, J. J. (1973). The effects of cued orienting tasks on the free recall of words. Journal of Verbal Learning and Verbal Behavior, 12, 489-498. http://dx.doi.org/10.1016/S0022-5371(73)80029-5

Von Restorff, H. (1933). Über die Wirkung von Bereichsbildungen im Spurenfeld (The effects of field formation in the trace field). Psychologie Forschung, 18, 299-34.

Wallace, W. P. (1965). Review of the historical, empirical, and the theoretical status of the Von Restorff phenomenon. Psychological Bulletin, 63, 410-424. http://dx.doi.org/10.1037/h0022001

Walsh, D. A., \& Jenkins, J. J. (1973). Effects of orienting tasks on free recall in incidental learning: "Difficulty", 
"Effort", and "Process" explanations. Journal of Verbal Learning and Verbal Behavior, 12, 481-488. http://dx.doi.org/10.1016/S0022-5371(73)80028-3

Watkins, O. C., \& Watkins, M. J. (1975). Buildup of proactive inhibition as a cue-overload effect. Journal of Experimental Psychology: Human Learning and Memory, 104, 442-452. http://dx.doi.org/10.1037/0278-7393.1.4.442 